\title{
SODART TELESCOPE ON SPECTRUM-RÖNTGEN-GAMMA AND ITS INSTRUMENTATION
}

\author{
HERBERT W. SCHNOPPER \\ Danish Space Research Institute, Gl. Lundtoftevej 7, DK-2800 Lyngby, Denmark
}

\begin{abstract}
SPECTRUM-RÖNTGEN-GAMMA (SRG) is one of a new series of large astronomical missions being planned by the Soviet Union and is scheduled for launch in mid-1993. The Space Research Institute (IKI) of the Academy of Sciences of the USSR and the Babakin Center (BC) are responsible for the scientific supervision and spacecraft construction, respectively. Mission objectives include broad and narrow band imaging spectroscopy over a wide range of energies from EUV through gamma rays with particular emphasis on extragalactic objects. The design of the Soviet Danish Röntgen Telescope (SODART) consists of two thin foil, conical shell approximations to Wolter 1 geometry. The reflectors are rolled aluminum foils which have been dipped in acrylic lacquer and coated with gold resulting in a super smooth surface. Each telescope has an aperture of $60 \mathrm{~cm}$, a focal length of $8 \mathrm{~m}$, a field of view of $1 \mathrm{deg}$ and is designed to have a halfpower width of $\leq 2$ arcmin. The conical geometry contributes 15 arcsec and manufacturing tolerances in the support structure and the quality of the figure of the foil the rest. The contribution from X-ray scattering is insignificant. Focal plane slides can position one of four instruments at the focus of each telescope. Images and spectra will be recorded with position sensitive proportional counters with spectral resolution as good as $13 \%$ at $6 \mathrm{keV}$. Spectral resolution of $2.5 \%$ at $6 \mathrm{keV}$ is provided by an array of 19 cooled silicon detectors. A broad band polarimeter will be sensitive to residual polarization as low as $1 \%$. An objective Bragg crystal panel, placed in front of one of the telescopes, will be capable of high resolution spectroscopic studies $((E / \Delta E)) \sim 1000)$ of point- and extended sources.
\end{abstract}

\section{Introduction}

SRG is to be the first of a series of new astronomical observatories to be launched by the Soviet Union under the sponsorship of the Academy of Sciences of the USSR. The expected launch date is mid-1993 and the observatory is expected to be operated for a period of 5 years. The satellite will be launched into a deep, highly eccentric orbit with a period of about four days from which long duration observations can be made. With all the operational constraints taken into account, there is access to about 80 per cent of the celestial sphere at any time. The major facility on board is the SODART X-ray telescope and its associated focal plane instruments. Instrumentation in addition to SODART will cover the energy range from EUV to gamma rays. A complete description of the SRG system (Figure 1) will be found in the paper presented by R. Sunyaev (1990) at this Colloquium.

\section{SODART}

The system consists of X-ray optics, thermal and structural elements, focal plane instruments, a focal plane transport assembly and an objective crystal spectrometer 


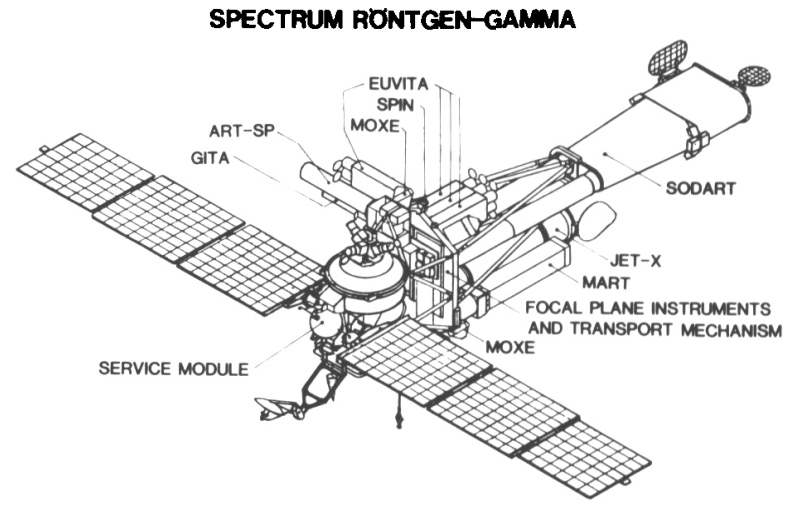

Fig. 1. SPECTRUM-RÖNTGEN-GAMMA (R.S. Kremnev, Babakin Center.)

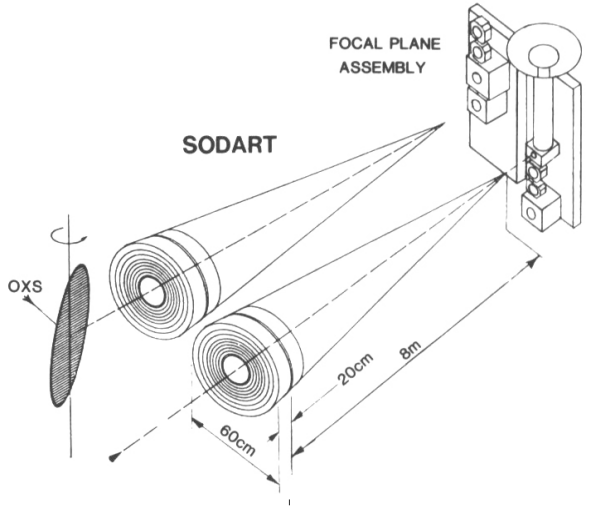

Fig. 2. The telescopes and instruments of SODART.

(Figure 2). The elements of SODART provided by DSRI are called XSPECT. Brief descriptions of these elements follow.

\subsection{X-RAY OPTICS}

Serlemitsos, Petri, Glasser, and Birsa (1984) pointed out the advantages of using thin foil, conical approximations to Wolter 1 optics. A large fraction of the available aperture can be filled by densely nesting many shells of thin foils yielding a large collecting area and an extended energy range. The design goal for the XSPECT telescope is a Half-Power Width (HPW) of $<2$ arcmin of which $<15$ arcsec comes from the conical geometry. Sheets of commercially available rolled aluminum foil, $0.3 \mathrm{~mm}$ thick are the raw material for the telescopes. After an initial inspection, the foils are cut to size, chemically cleaned, rolled to an approximate radius of curvature, coated with a thin uniform layer of acrylic lacquer and, finally, a thin layer of gold is evaporated onto the surface. The parameters chosen for the XSPECT telescope are listed in Table I (Westergaard et al., 1990). Two such telescopes are to be provided 
TABLE I

XSPECT conical approximation telescopes

\begin{tabular}{lc}
\hline & Field of view \\
Half-power width & $60 \mathrm{arcmin}$ \\
Focal length & $<2$ arcmin \\
Diameter & $8 \mathrm{~m}$ \\
Reflecting surface & $60 \mathrm{~cm}$ \\
Effective area & $500 \AA \mathrm{Agld}$ \\
& $1700 \mathrm{~cm}^{2} @ 2 \mathrm{keV}$ \\
& $1200 \mathrm{~cm}^{2} @ 8 \mathrm{keV}$ \\
& $60 \mathrm{~cm}^{2} @ 20 \mathrm{keV}$ \\
Shell material & $0.3 \mathrm{~mm}$ aluminum \\
Outer shell diameter & $60 \mathrm{~cm}$ \\
Inner shell diameter & $16 \mathrm{~cm}$ \\
Shell separation (min) & $0.5 \mathrm{~mm}$ \\
Shell length & $20 \mathrm{~cm}$ \\
Number of shells & 154 \\
Mass per telescope & $90 \mathrm{~kg}$ \\
Total mirrored surface & $75 \mathrm{~m}$ \\
\hline
\end{tabular}
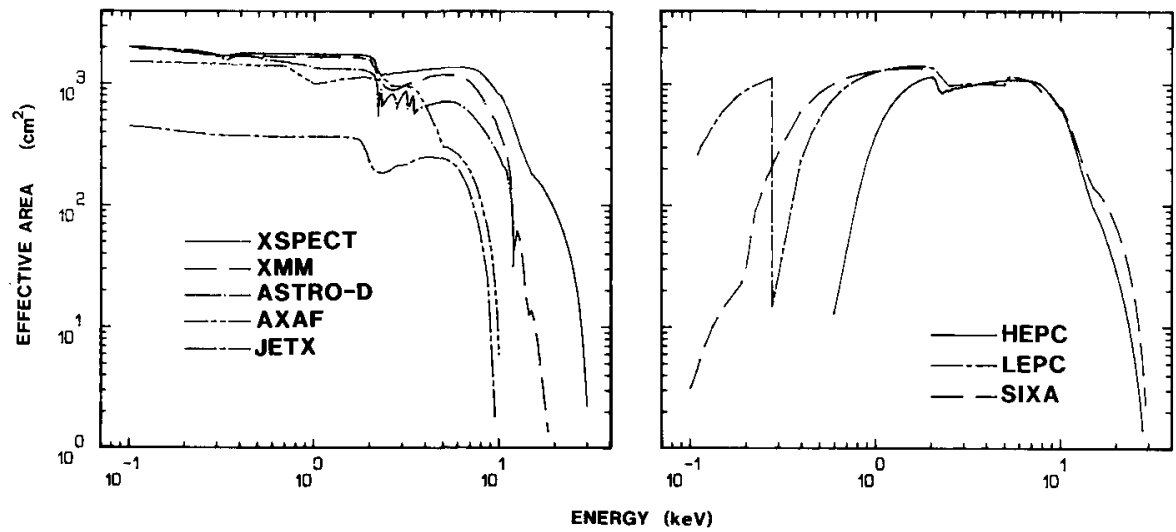

Fig. 3. Left: On-axis collecting area for various telescopes. Right: XSPECT's effective area for various detectors (N.J. Westergaard, DSRI).

for SODART. Figure 3 shows the collecting area which can be expected for a single telescope. Curves for other telescopes are shown for comparison. Measurements of reflection efficiencies at various X-ray energies for foil samples prepared at DSRI have been found to agree with the theoretical values which have been used to construct the XSPECT curve.

Each XSPECT telescope consists of two sections, 1 alpha and 3 alpha, each of which is divided into quadrants (Figure 4). After assembly, each quadrant is mounted to a reference hub whose axis is made accurately parallel to the axis of a well collimated optical beam $25 \mathrm{~cm}$ in diameter. The reflected beam can be recorded by a CCD camera which can be placed at any distance along the converging beam. These optical test are used to control the adjustments of the ribs which guide the foils into the optimum position. Diffraction makes it impossible to measure the HPW directly by this method. When, however, a slit is used to scan the quadrant it is possible to measure the centroid of the image focused by the small portion 
of the illuminated foils. The distribution of these centroids is a good indication of the contribution to the HPW which comes from manufacturing tolerances and misalignment. Recent measurements on an assembled, but not aligned quadrant show that mechanical errors are well within the goal of HPW $<2$ arc min.

Once the alignment procedure is completed, the $\mathrm{X}$-ray reflecting properties, including the HPW, will be measured in a specially constructed facility. A pencil beam from a high power, rotating anode X-ray tube is made highly parallel and monochromatic by perfect silicon crystals. The quadrant under test (and later the completed telescope) is mounted on a translation and rotation stage (rotation around the optical axis). An imaging proportional counter is placed just after the telescope and a raster scan of the beam is made for each offset angle to simulate the on-and off-axis behavior of the telescope. The synthesized full beam image at the correct focal distance can be constructed by computer processing. The anode material is replaceable to allow tests at several energies. Work is in progress to prepare a full aperture beam test using the synchrotron radiation facility at Daresbury (Lewis et al., 1990). Final calibration will take place in orbit.

\subsection{FOCAL PLANE DETECTORS}

\subsubsection{XSPECT MicroStrip Proportional Counters (MSPC)}

In conventional proportional counters the electron avalanche takes place in the high field region defined by anode and cathode grids made from finely spaced wires. These designs usually lead to gain and position non-linearity, count rate restrictions and high operating voltages. The MSPC is a novel approach, in which the wire grids are replaced by narrowly spaced, conducting microstrips (Oed, 1988; Budtz-Jörgensen et al., 1989) which are accurately deposited $( \pm 2 \mu \mathrm{m})$ on an insulating substrate. The XSPECT MSPC detector based on these principles is shown in Figure 5. A highand a low-energy (HEPC and LEPC) detector will be provided for each telescope. The parameters chosen for them are listed in Table II.

$\mathrm{X}$-direction positions are obtained from the charge induced on the wire grid which is suspended above the microstrip. The wires are connected with resistors and the charge division is read out by suitable spaced preamplifiers that insure the appropriate position sensitivity. Y-direction positions are obtained from the cathode strips on the microstrip which are connected by a resistive strip on the substrate. Energy, event risetime, and anti-coincidence discrimination are used to reject background. Results from energy- and position-resolution, and pulse-shape background rejection measurements are shown in Figure 6.

\subsubsection{Other focal plane detectors}

Two imaging Focal Plane Röntgen Detectors (FRD) described by Sunyaev (1990), a Silicon X-ray Array (SIXA) described by Vilhu (1990) and a Stellar X-Ray Polarimeter (SXRP) (Kaaret et al., 1989) complete the complement of focal plane instruments. They are shown in place on the focal plane transport assembly (Figure 7). 

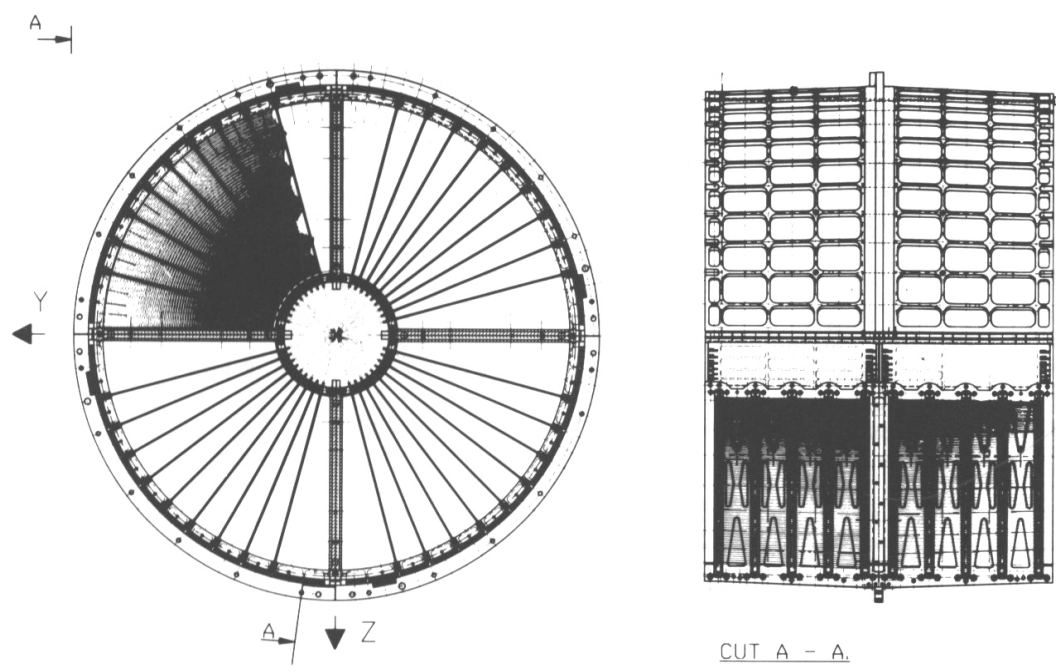

CUT A - A.

Fig. 4. Structure of the XSPECT telescope (J. Polny, DSRI).

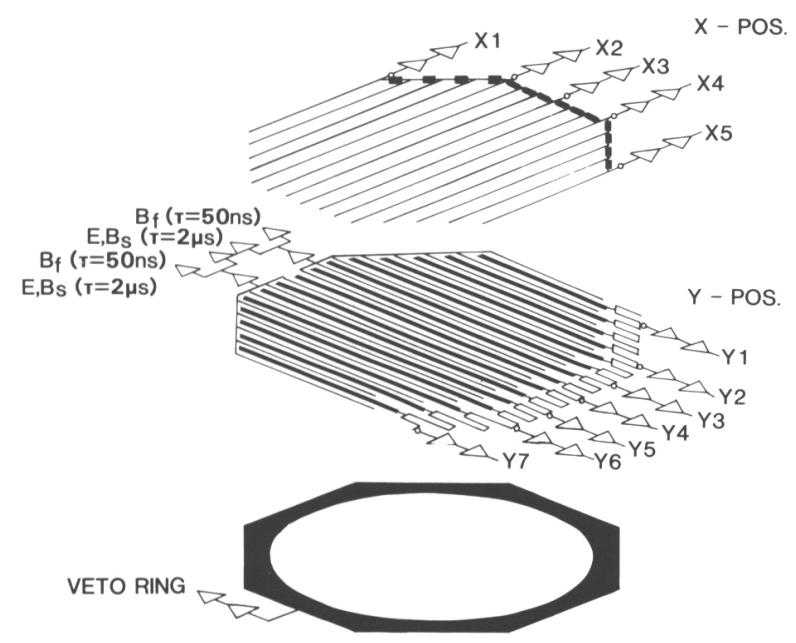

Fig. 5. The XSPECT MSPC (C. Budtz-Jörgensen). 
TABLE II

Proportional Counter Parameters

Field of view

Diameter of active area

Diameter of window

Position resolution

Time resolution

Background rejection

Total memory

Gas

Energy resolution
60 arcmin

$160 \mathrm{~mm}$

$140 \mathrm{~mm}$

$<1 \mathrm{~mm}$

$<5 \mu \mathrm{s}$

$>99$ per cent

128 Mbytes

Xe $90 \%$, Propylene $10 \%$

$0.32 \mathrm{E}^{1} \mathrm{keV}$

Energy range
Dynamic range
Efficiency
Gas thickness
Gas pressure
Window

$\begin{array}{ll}\text { HEPC } & \text { LEPC } \\ 2-25 & 0.5-8 \mathrm{keV} \\ 625 & 800 \\ >0.7,2<\mathrm{E}<15 & >0.7,1<\mathrm{E}<8 \mathrm{keV} \\ 4 & 2 \mathrm{~cm} \\ 1 & 0.5 \mathrm{~atm} \\ 13 \mu \mathrm{m} \mathrm{Be} & 0.5 \mu \mathrm{m} \text { plastic } \\ & 0.2 \mu \mathrm{m} \text { diamond } \\ & 0.02 \mu \mathrm{m} \mathrm{Al}\end{array}$

\subsection{XSPECT Objective Crystal Spectrometer (OXS)}

The OXS concept separates the processes of energy dispersion and imaging (Schnopper and Byrnak, 1987). The large mosaic panel of flat crystals in front of the telescope acts as a narrow pass filter and a mirror. Each pixel in the reflected field of view of the telescope satisfies a specific Bragg angle on the crystal and, therefore, each pixel in the detector can be identified with a particular energy. Scans which involve repositioning of the telescope axis and the angle between the crystal panel and the telescope axis $(45+15 /-10 \mathrm{deg})$ yield either the spectrum of a point source or energy resolved images of an extended source. Parameters chosen for OXS are given in Table III. It is intended that MultiLayer (ML) structures be deposited on the surfaces of the LiF and Ge crystals to allow simultaneous measurements of the $\mathrm{Fe}, \mathrm{S}$ and $\mathrm{O}$ energy ranges. The use of both 1 st and 2nd order ML reflections will allow studies of line emission at energies below the $\mathrm{C}$ K-edge cut-off imposed by the entrance window (Christensen et al., 1990).

\subsection{THERMAL AND STRUCTURAL ELEMENTS}

SODART is launched in a folded configuration which just fits inside the shroud attached to the PROTON launcher. SODART is then deployed in orbit and locks into position for observing within the carbon fiber epoxy structure shown in Figure 1. Doors at the lower end of the telescope thermal enclosure open and telescoping tubes are lowered to make contact with the focal plane transport assembly. Heaters and thermal baffles maintain the appropriate temperature conditions for the telescopes and the crystal panel.

Each of the telescope assemblies will be provided with a laser ranging system which will measure displacements in $\mathrm{x}, \mathrm{y}$ and $\mathrm{z}$ with respect to the intersection of the telescope axis with the ideal focal plane. The tilt of the telescope axis with 
TABLE III

Crystals for the XSPECT OXS

\begin{tabular}{|c|c|c|c|c|c|c|}
\hline & Crystal & Plane & $2 d(A)$ & Range (keV) & $R_{c}(\mathrm{rad})$ & $\begin{array}{l}E / \Delta E \\
(35<\theta<60 \mathrm{deg})\end{array}$ \\
\hline \multirow[t]{2}{*}{ Side 1} & $\mathrm{LiF}$ & $(220)$ & 2.848 & $5.00-7.40$ & $1.7 \times 10^{-4}$ & $1375-2300$ \\
\hline & $\begin{array}{l}\mathrm{ML} \\
\mathrm{Ni} / \mathrm{C} \text { on } \\
\mathrm{LiF}(220)\end{array}$ & $\begin{array}{l}(001) \\
(002)\end{array}$ & $\begin{array}{l}67 \\
67\end{array}$ & $\begin{array}{l}0.21-0.31 \\
0.43-0.63\end{array}$ & $\begin{array}{l}4.0 \times 10^{-3} \\
1.6 \times 10^{-4}\end{array}$ & $\begin{array}{r}70 \\
300\end{array}$ \\
\hline or, & $\begin{array}{l}\mathrm{ML} \\
\mathrm{Ni} / \mathrm{C} \text { or } \mathrm{W} / \mathrm{B}_{4} \mathrm{C} \\
\text { on } \mathrm{LiF}(220)\end{array}$ & $\begin{array}{l}(001) \\
(002)\end{array}$ & $\begin{array}{l}39.2 \\
39.2\end{array}$ & $\begin{array}{l}0.42-0.62 \\
0.84-1.23\end{array}$ & $\begin{array}{l}4.0 \times 10^{-4} \\
1.0 \times 10^{-4}\end{array}$ & $\begin{array}{l}300 \\
400\end{array}$ \\
\hline \multirow[t]{3}{*}{ Side 2} & $\mathrm{Ge}$ & (111) & 6.532 & $2.19-3.23$ & $2.9 \times 10^{-4}$ & 2575 \\
\hline & RAP & $(001)$ & 26.121 & $0.55-0.81$ & $5.0 \times 10^{-5}$ & 770 \\
\hline & $\begin{array}{l}\mathrm{ML} \\
\mathrm{Ni} / \mathrm{C} \text { on } \\
\text { on Ge (111) }\end{array}$ & $\begin{array}{l}(001) \\
(002)\end{array}$ & $\begin{array}{l}56 \\
56\end{array}$ & $\begin{array}{l}0.26-0.38 \\
0.51-0.75\end{array}$ & $\begin{array}{l}4.3 \times 10^{-3} \\
2.0 \times 10^{-3}\end{array}$ & $\begin{array}{r}80 \\
300\end{array}$ \\
\hline
\end{tabular}

respect to the line perpendicular to the focal plane will also be measured. These measurements will be correlated with 3 -axis aspect information derived from star sensors mounted on the fixed portion of the focal plane structure. The possibility of a star sensor mounted on the telescope structure is being considered. BC is responsible for these elements of SODART. They also provide the spacecraft and integrate the payload. IKI provides the overall project management.

\section{Sensitivities}

The sensitivity to emission lines of a single module in combination with a gas detector has been calculated with the parameters given in the Table IV. The iron line at $\sim 6.7 \mathrm{keV}$ is a blend of relatively few lines whereas the oxygen lines around $0.7 \mathrm{keV}$ are surrounded by many other lines which makes the continuum determination difficult.

The points in Figure 8 are AGN from the Turner and Punds (1989) EXOSAT sample. The equivalent widths are assumed and used to indicate the typical times required to observe the lines. The continuum flux has been evaluated from the reported luminosity and power-law slope.

\section{Acknowledgements}

The staff at DSRI, IKI and BC have made significant contributions to this work and $I$ thank them warmly for their assistance in preparing the manuscript. 

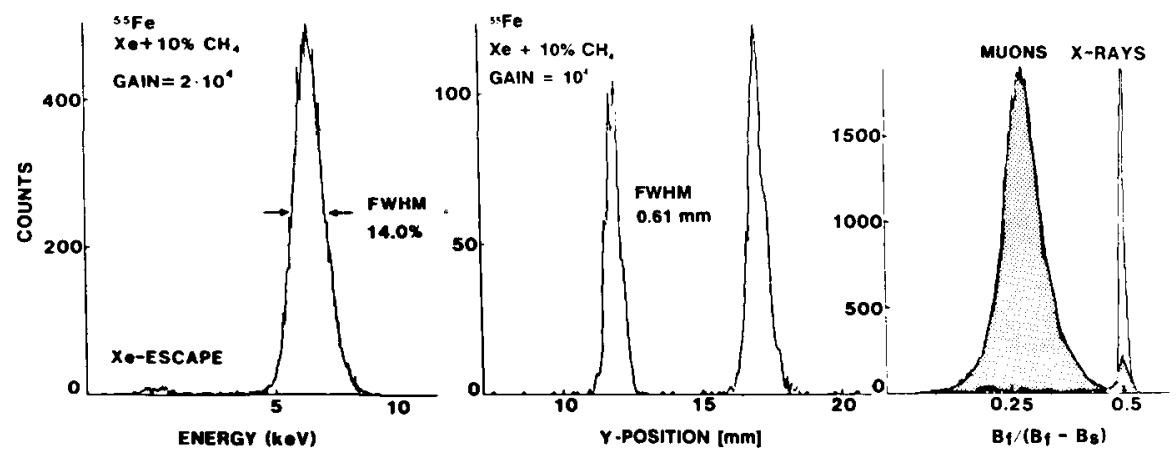

Fig. 6. Energy, position and pulse shape background rejection resolution with a breadboard MSPC (C. Budtz-Jörgensen, DSRI).

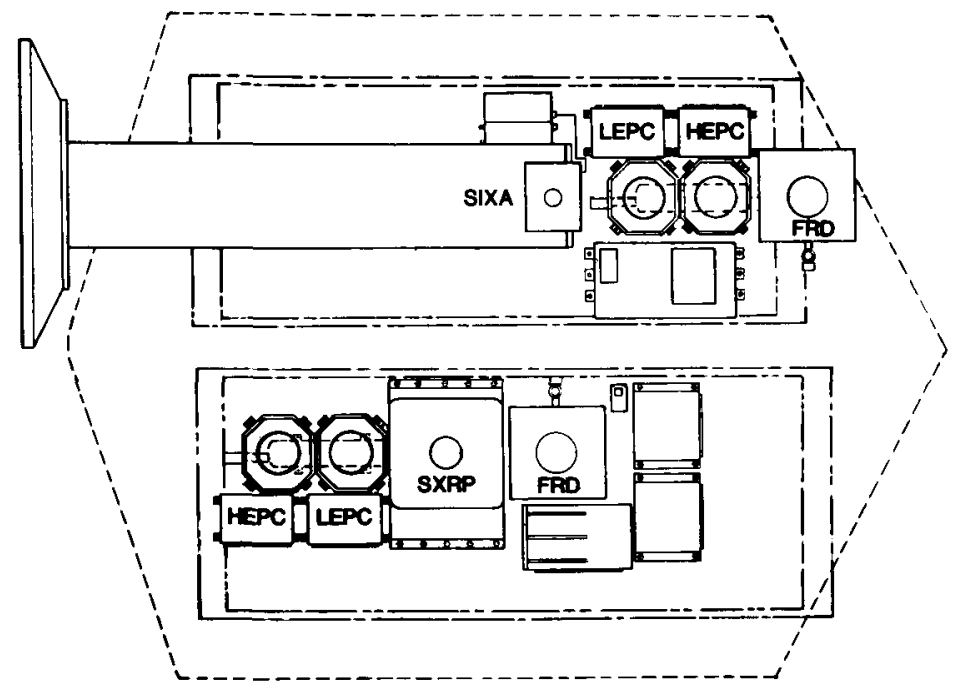

Fig. 7. The SODART focal plane assembly. 
IRON LINE DETECTION

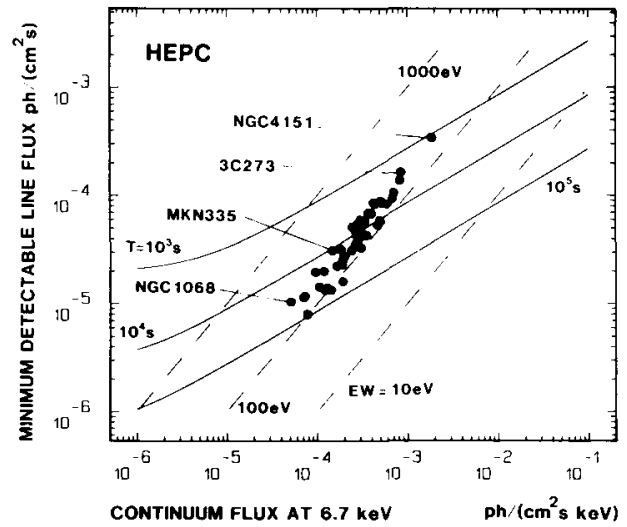

\section{OXYGEN LINE DETECTION}

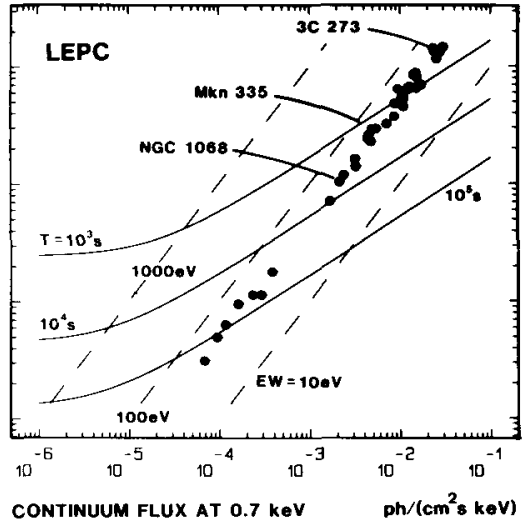

Fig. 8. Minimum detectable iron and oxygen line fluxes for various observing times and line equivalent widths (EW). The AGNs shown have all been assigned the same $\mathrm{EW}(150 \mathrm{eV}$ iron and $50 \mathrm{eV}$ for oxygen). 
TABLE IV

Parameters used for Line Sensitivity Calculations

\begin{tabular}{|c|c|c|c|}
\hline & Oxygen in LEPC & Iron in HEPC & \\
\hline $\begin{array}{l}\text { Line energy } \\
\text { Telescope eff. area } \\
\text { Detector quant. eff. } \\
\text { Energy resolution } \\
\text { Particle background } \\
\text { Search cell size } \\
\text { Fraction in cell }\end{array}$ & $\begin{array}{c}0.7 \\
1766 \\
0.54 \\
0.27 \\
10^{-3} \\
0.22 \\
0.5\end{array}$ & $\begin{array}{c}6.7 \\
1356 \\
0.80 \\
0.80 \\
10^{-3} \\
0.22 \\
0.5\end{array}$ & $\begin{array}{l}\mathrm{keV} \\
\mathrm{cm}^{2} \\
\mathrm{keV} \\
\mathrm{cts} \mathrm{cm}^{-2} \mathrm{~s}^{-1} \mathrm{keV}^{-1} \\
\mathrm{~cm}^{2}\end{array}$ \\
\hline
\end{tabular}

\section{References}

Budtz-Joergensen, C., Madsen, M.M., Jonasson, P., Schnopper, H.W., and Oed, A.: 1989, SPIE Proceedings, 982

Christensen, F.E.: 1990, SPIE Proceedings, in press

Kaaret, P. et al.: 1989, SPIE Proceedings, 1160

Lewis, R., Bordas, J., and Christensen, F.E.: 1990, Opt. Eng., in press

Oed, A.: 1988, Nucl. Instr. Meth. A263, 351-359

Schnopper, H.W., and Byrnak, B.P.: 1987, Appl. Opt. 26, 2871

Serlemitsos, P.J., Petre, R., Glasser, C., and Birsa, F.: 1984, IEEE Trans. Nucl. Sci. NS-31, 786

Sunyaev, R.: 1991, IAU Colloquium 123, Kluwer Academic Publishers, Dordrecht

Turner, T.J. and Pounds, K.A.: 1989, Mon. Not. R. Astr. Soc. 240, 833

Vilhu, O.: 1991, IAU Colloquium 123, Kluwer Academic Publishers, Dordrecht

Westergaard, N.-J., et al.: 1990, Opt. Eng., in press 